\title{
Psicooncología
}

ISSN: $1696-7240$

\section{Validación de la versión castellana del Inventario de Fatalismo de Powe en población mexicana con cáncer}

\author{
Rosa Lilia Castillo-López ${ }^{1}$, Roberto Lagunes-Córdoba², Oscar Galindo Vázquez ${ }^{3}$, Edgar \\ Landa-Ramírez ${ }^{4}$ y María Luisa Marván-Garduño ${ }^{5 *}$
}

Resumen: Objetivo: validar la versión en español del Inventario de Fatalismo de Powe (SPFI) en población mexicana con cáncer. Método: Se aplicó el SPFI a 133 mujeres con cáncer de mama. Se realizó un análisis factorial con rotación Oblimin. Posteriormente se evaluó la consistencia interna con el coeficiente Alfa de Cronbach y por último se realizaron análisis de correlación entre los factores del cuestionario. El instrumento resultante se nombró Inventario de Fatalismo de Powe en Español-Cáncer (IFPE-C).Resultados: Se eliminaron siete de los 15 reactivos originales y se conservaron ocho, todos ellos con cargas factoriales superiores a 0,40 . Se obtuvo una estructura de tres factores que explicaron el $73,7 \%$ de la varianza y se llamaron: Inutilidad del tratamiento con un $\alpha=0,85$, Predestinación con un $\alpha=0,918$ y Pensamientos de muerte con un $\alpha=0,73$. La consistencia interna para la escala global fue de $\alpha=0,80$. Se obtuvieron correlaciones estadísticamente significativas entre los factores. Conclusión: el IFPE-C demostró ser un instrumento valido y confiable, que puede ser utilizado tanto en entornos clínicos como de investigación para identificar las creencias fatalistas acerca del cáncer de los pacientes. Palabras clave: fatalismo, Inventario de Fatalismo de Powe, validación, cáncer.

\section{[en] Validation of the Castilian version of Powe's Fatalism Inventory in a Mexican population with cancer}

Abstract: Objective: To validate the Spanish version of Powe's Fatalism Inventory (SPFI) in Mexican
population with cancer.Method: The SPFI was applied to 133 women with breast cancer. A factorial
analysis with Oblimin rotation was conducted, Then, the internal consistency was evaluated with the

1 Rosa Lilia Castillo-López. Universidad Veracruzana. Instituto de Investigaciones Psicológicas. México.

E-mail: psic.roxcastillo@gmail.com

2 Roberto Lagunes-Córdoba Universidad Veracruzana. Instituto de Investigaciones Psicológicas. México.

E-mail: rlc.academico@yahoo.com.mx

3 Oscar Galindo Vázquez. Servicio de Psico-oncología, Instituto Nacional de Cancerología. Av. San Fernando 22. Sección XVI, Tlalpan, 14080, Ciudad de México. México.

E-mail: psigalindo@yahoo.com.mx

4 Edgar Landa-Ramírez Hospital General "Dr. Manuel Gea González". Programa de Psicología Urgencias/División de Urgencias y Observación. Universidad Nacional Autónoma de México (UNAM). Facultad de Psicología/Programa de Psicología de la Salud y Medicina Conductual. México.

E-mail: edgar_landa_ramirez@yahoo.com.mx

5 María Luisa Marván Instituto de Investigaciones Psicológicas Universidad Veracruzana. México.

E-mail: mlmarvan@gmail.com

* Dirección de correspondencia: Dra. María Luisa Marván Instituto de Investigaciones Psicológicas Universidad Veracruzana Av. Dr. Luis Castelazo Ayala s/n Col. Industrial Ánimas. C.P. 91190 Xalapa, Ver. México. E-mail: mlmarvan@gmail.com 
Cronbach's Alpha coefficient and finally, correlation analyses among the questionnaire factors were conducted. The final instrument was named Spanish Powe Fatalism Inventory-Cancer (IFPE-C). Results: Seven of the original 15 items were eliminated and eight were retained, all with factor loads greater than 0.40 . We obtained a structure of three factors that explained $73.7 \%$ of the variance and were called: Uselessness of the treatment with $\alpha=0.85$, Predestination with $\alpha=0.918$ and Thoughts of death with $\alpha=0.73$. The internal consistency for the global scale was $\alpha=0.80$. Statistically significant correlations were obtained between the factors. Conclusion: the IFPE-C proved to be a valid and reliable instrument that can be used both in clinical and research settings to identify fatalistic beliefs about cancer of patients.

Keywords: fatalism, Powe Fatalism Inventory, validation, cancer.

Sumario. 1. Introducción 2. Método 2.1. Participantes 2.2. Instrumento 2.3. Procedimiento 2.4. Análisis estadístico 3. Resultados 4. Discusión 5. Referencias bibliográficas.

Cómo citar: Castillo-López RL, Lagunes-Córdoba R, Galindo Vázquez O, Landa-Ramírez E, MarvánGarduño ML. Validación de la versión castellana del Inventario de Fatalismo de Powe en población mexicana con cáncer. Psicooncología 2019; 16(1): 89-99. doi: 10.5209/PSIC.63650.

\section{Introducción}

Existen diversas barreras psicosociales que obstaculizan las conductas saludables relacionadas con la prevención, diagnóstico oportuno, y adherencia al tratamiento del cáncer, entre las que se encuentran las creencias fatalistas ${ }^{(1-4)}$. El concepto de fatalismo es definido como la creencia general de que el curso del destino no puede ser cambiado, y que los eventos de la vida están más allá del control de la persona ${ }^{(5)}$. Powe definió el fatalismo acerca del cáncer como la idea de que la presencia de esta enfermedad está predeterminada y que además, una vez diagnosticado, el cáncer provocará inevitablemente la muerte de quien lo padece ${ }^{(6)}$. Desde esta perspectiva, el fatalismo acerca del cáncer tiene un papel crucial que se enfoca en el temor, la impotencia y la desesperanza con respecto a este padecimiento; esto implica que una persona fatalista perciba el cáncer como una lucha inútil debido a la imposibilidad de superarlo, ya que lo relaciona con la muerte ${ }^{(7)}$. Este constructo ha sido explorado para entender algunos aspectos psicológicos relacionados con el cáncer en sus diversas fases, desde la prevención hasta la sobrevivencia ${ }^{(3,8,9)}$.

El fatalismo acerca del cáncer está fuertemente influenciado por diversos factores de la cultura en la que se desenvuelve el individuo ${ }^{(3,8)}$. En general, las personas de niveles socioeconómicos bajos tienden a mostrar más creencias fatalistas ${ }^{(10)}$. En estudios realizados con mujeres en Estados Unidos se ha encontrado que las mujeres latinas reportan más creencias fatalistas que las no latinas ${ }^{(11,12)}$ y de hecho, se ha concluido que el fatalismo es un componente importante de la cultura relacionada con la salud en la población latina ${ }^{(5,13)}$.

Existen algunos instrumentos para medir el fatalismo en el área de la salud ${ }^{(14)}$. Sin embargo, existe la limitación de que no son específicos para contextos oncológicos. Hasta donde se sabe, solo existe un instrumento para medir los niveles de fatalismo acerca del cáncer, que es el Inventario de Fatalismo de Powe (PFI por sus siglas en inglés), instrumento que se desarrolló en EEUU( ${ }^{(7)}$. El PFI está basado en el Modelo de Fatalismo de Powe que incluye cuatro componentes: 1) "Miedo", que se refiere a la sensación de temor con respecto a la presencia del cáncer, 2) "Predeterminación", que es la creencia de que la enfermedad ya estaba establecida por fuerzas externas al 
individuo, 3) "Pesimismo", que implica un enfoque centrado en los aspectos negativos del cáncer, 4) "Inevitabilidad de la muerte", que se refiere a la creencia de que la enfermedad provocará la muerte de quien lo padece, sin que el individuo pueda hacer algo para prevenirla ${ }^{(6)}$. El modelo fue desarrollado en el contexto de la población afroamericana, y el PFI se creó justamente con esa población, específicamente con personas sin diagnóstico de cáncer. Los reactivos del PFI se agrupan en cuatro factores que corresponden a los cuatro componentes del modelo teórico de base, y explican el $59 \%$ de la varianza, con una consistencia interna de $0,87^{(6)}$.

Se han hecho algunas criticas al PFI, ya que los cuatro componentes del constructo fatalismo parecen no estar reflejados de forma adecuada, pues algunos de ellos parecen superponerse entre si, y otros, como el miedo, parecen ser mas una consecuencia del fatalismo que un componente de éste. Lo anterior ha significado una critica a la multidemensionalidad de la escala, pues se cree que tres (inevitabilidad de la muerte, pesimismo y predeterminación) de los cuatro componentes podrían conformar un solo factor que represente de forma mas clara el fatalismo, al menos al respecto del cáncer ${ }^{(14)}$. A pesar de ello, diversos autores han utilizado el PFI en población sin cáncer y han encontrado que el fatalismo se asocia con variables como la evitación de información sobre el cáncer ${ }^{(15,16)}$, o la poca conciencia sobre este padecimiento ${ }^{(17,18)}$. Sin embargo, es indispensable tomar en cuenta que en pacientes ya diagnosticados con cáncer, la percepción y creencias sobre esta enfermedad pueden ser distintas a las del resto de la población. En este sentido, existen algunos estudios en los que se han explorado las creencias fatalistas en pacientes con cáncer, usando ya sea el PFI completo o algunos de sus reactivos. En dichos estudios se ha encontrado que los pacientes presentan niveles elevados de fatalismo ${ }^{(19,20)}$, y que éste se asocia con algunos aspectos desapatativos como la baja autoeficacia, y además es una variable predictiva de la no adherencia al tratamiento oncológico ${ }^{(3,21)}$. Sin embargo, hasta donde se ha reportado, las creencias fatalistas no han sido exploradas en población diagnosticada con cáncer en países hispano parlantes, lo cual resulta indispensable si tomamos en cuenta la presencia del fatalismo en personas sin cáncer que radican en países de habla hispana.

ElPFI fue traducido al español y adaptado a la cultura méxico-americana con personas que vivían en la frontera entre EEUU y México, pero éstas no tenían diagnóstico de cáncer. El instrumento fue denominado Spanish Powe Fatalism Inventory (SPFI) ${ }^{(22)}$. Debido a que no se cuenta con un instrumento en español, valido y confiable para evaluar el fatalismo en población oncológica, el objetivo de la presente investigación fue validar el SPFI en personas mexicanas diagnosticadas con cáncer, en particular con cáncer de mama (CaMa) debido a que éste es el cáncer con mayor incidencia y prevalencia a nivel mundial ${ }^{(23)}$. Se decidió traducir el nombre del cuestionario al español y agregarle la palabra cáncer; de tal manera que el nombre propuesto del cuestionario resultante es Inventario de Fatalismo de Powe en Español-Cáncer (IFPE-C).

\section{Método}

\subsection{Participantes}

Las participantes fueron mujeres con CaMa que estaban siendo atendidas en el Instituto Nacional de Cancerología (INCan), el cual cuenta con un hospital de alta 
especialidad y se ubica en la Ciudad de México. Los criterios de inclusión fueron: tener diagnóstico de CaMa en estadios in situ, I, II o III, y ser mayor de 18 años (mayoría de edad en México). Los criterios de exclusión fueron: ser paciente con recurrencia de la enfermedad, tener diagnóstico de cáncer de un segundo tumor primario, o tener algún déficit cognitivo que impidiera la comprensión del instrumento, o haber recibido atención psicológica inmediatamente después del diagnóstico. Este último criterio se estableció porque después de la aplicación de los instrumentos las pacientes recibieron atención psicológica en caso de requerirla. Como criterio de eliminación se consideró no responder la totalidad del instrumento.

Se obtuvieron datos de 133 pacientes cuya edad promedio fue de 51,5 años (desviación estándar $[\mathrm{DE}]=12,6)$. La mayoría de las participantes tenían hijos, prácticamente la mitad estaban casadas y se dedicaban a las labores del hogar. Aproximadamente la mitad habían estudiado educación media (12 años de estudios) o tenían estudios universitarios. Poco más de la mitad de las pacientes se encontraban en el estadio clínico II; aproximadamente una cuarta parte estaban en el estadio III, y el grupo mas pequeño fue el que estaba ya sea en el estadio 0 o en el I (ver tabla 1 ).

\subsection{Instrumento}

Se aplicaron preguntas cerradas sobre datos sociodemográficos y el SPFI, el cual consta de 15 reactivos con respuestas dicotómicas (si/no). Dichos reactivos, al igual que el original que está en inglés, también se agrupan en cuatro factores que corresponden al Modelo de Fatalismo de Powe, y explican el 57\% de la varianza, con una consistencia interna adecuada (alfa de Cronbach $=0,81$ ). El factor "Predeterminación" consta de seis reactivos, el de "Pesimismo" de cinco reactivos, el de "Inevitabilidad de la muerte" de dos reactivos, y el de "Miedo" de otros dos reactivos. La instrucción que se dio a las pacientes para responder el instrumento fue la siguiente: "Por favor lea las siguientes afirmaciones, posteriormente marque la opción que considere mas adecuada para su situación. Recuerde que no hay respuestas correctas o incorrectas".

\subsection{Procedimiento}

La investigación fue aprobada por el INCan y forma parte del proyecto de "Cáncer de mama de Psicooncología" de dicho instituto. Las pacientes con reciente diagnóstico de CaMa que cumplieron con los criterios de inclusión, se ubicaron a través del servicio de Unidad Funcional de Tumores Mamarios del INCan. Se les invitó a participar en una investigación de psicooncología, en la que se les aplicarían algunos cuestionarios, cuyos resultados se utilizarían para fines exclusivos de la investigación y serían confidenciales. Posteriormente, se obtuvo el consentimiento informado de aquellas pacientes que aceptaron participar (86\%), y se procedió a explicarles el proceso de aplicación de los cuestionarios. En total se aplicaron tres cuestionarios, uno de ellos fue el SPFI. Las participantes respondieron los instrumentos en las salas de espera del servicio de Tumores Mamarios del INCan, en presencia de una encuestadora, quien estaba capacitada en temas psicooncológicos y entrenada para realizar el proceso de aplicación de los instrumentos. El tiempo promedio en el que las participantes respondieron los tres cuestionarios fue de 30 minutos, y la aplicación se llevó a cabo de forma individual y oral con el fin de asegurarse que 
todas las pacientes entendieran bien todos los reactivos. En todos los casos se aclaró que no había respuestas correctas ni incorrectas. Los datos se recolectaron entre abril y agosto de 2017.

Tabla 1. Descripción de las variables sociodemográficas y clínicas

\begin{tabular}{llll}
\hline & Frecuencia & Porcentaje \\
\hline Estado civil & & & \\
& Soltera & 32 & $24,1 \%$ \\
& Casada/unión libre & 68 & $51,1 \%$ \\
& Divorciada/separada & 21 & $15,8 \%$ \\
& Viuda & 12 & $9,0 \%$
\end{tabular}

Ocupación

$\begin{array}{lll}\text { Ama de casa } & 71 & 53,4 \% \\ \text { Empleada } & 27 & 20,3 \% \\ \text { Sin empleo } & 35 & 26,3 \%\end{array}$

Zona de residencia

$\begin{array}{lll}\text { Rural } & 35 & 26,3 \% \\ \text { Urbana } & 98 & 73,7 \%\end{array}$

Escolaridad

\begin{tabular}{lll} 
Analfabeta & 14 & $10,5 \%$ \\
Básica (9 años) & 53 & $39,8 \%$ \\
Media (12 años) & 36 & $27,1 \%$ \\
Superior (más de 12 años) & 30 & $22,6 \%$ \\
\hline \multicolumn{2}{l}{ Frecuencia } & Porcentaje \\
\hline Estadio clínico & & \\
0 y 1 & 23 & $17,3 \%$ \\
2 & 75 & $56,4 \%$ \\
3 & 35 & $26,3 \%$ \\
\hline
\end{tabular}

\subsection{Análisis estadístico}

Se analizó la distribución de los datos mediante la prueba de Kolmogórov-Smirnov, encontrando una distribución no normal. Se realizaron las pruebas de Esfericidad de Bartlett y la de Kaiser-Meyer-Olkin de adecuación de muestreo. Para establecer el poder discriminativo de los reactivos se siguió el método de grupos extremos, utilizando la prueba U de Mann-Whitney para comparar la puntuación total del 
cuestionario entre el $27 \%$ de las participantes que obtuvieron las puntuaciones más altas y el $27 \%$ que tuvieron las puntuaciones bajas ${ }^{(24)}$. Todos los valores de probabilidad $<0,05$ se consideraron significativos. Por último, se calcularon terciles para establecer los puntos de corte y poder establecer el nivel de fatalismo de las personas que contestaron el IFPE-C.

El análisis factorial se realizó mediante el procedimiento de extracción de mínimos cuadrados no ponderados, el cual se recomienda para datos con distribuciones no normales que no cumplen con los supuestos necesarios para el uso de la versión paramétrica del análisis factorial ${ }^{(25,26)}$, y se hizo una rotación Oblimin con normalización Kaiser. Este procedimiento se repitió tantas veces como fue necesario para obtener una estructura factorial estable, y se eliminaron los reactivos que tuvieron una carga factorial menor a 0,40. La consistencia interna se evaluó por medio del coeficiente Alfa de Cronbach para cada uno de los factores y para el cuestionario global. De acuerdo a Cronbach y Shavelson ${ }^{(27)}$, un valor de $\alpha \geq 0,70$ refleja una consistencia interna aceptable, $\geq 0,80$ alta $y \geq 0,90$ muy alta. También se realizaron correlaciones de Pearson entre los factores resultantes del cuestionario. Finalmente, se calcularon los terciles del cuestionario resultante para establecer los puntos de corte de las puntuaciones altas, intermedias y bajas.

\section{Resultados}

Se realizó el análisis de grupos extremos, el cual mostró que todos los reactivos tenían una capacidad discriminatoria adecuada, pues las diferencias entre los participantes que obtuvieron los puntajes más altos y los que obtuvieron los puntajes más bajos en el cuestionario fueron significativas.

Se determinó la factibilidad del análisis factorial exploratorio calculando el índice de adecuación muestral Kaiser-Meyer-Olkin $(\mathrm{KMO}=0,73)$ y la prueba de esfericidad de Bartlett $\left(X^{2}=593,68 ; \mathrm{gl}=28 ; \mathrm{p}<0,001\right)$, observándose una intercorrelación adecuada para realizar el análisis.

Se realizaron cuatro rondas de análisis factorial exploratorio con una rotación Oblimin. En la primera se eliminaron tres reactivos y en la segunda se eliminó otro, todos ellos debido a que presentaban cargas factoriales inferiores a 0,40 . En la tercera ronda todos los reactivos tuvieron cargas factoriales aceptables, y la regla Kaiser de autovalores superiores a 1 sugirió la extracción de 4 factores. No obstante, uno de los factores, compuesto por tres reactivos (sobre la relación entre la alimentación y el cáncer) no tenía congruencia con el constructo fatalismo en el contexto de la población oncológica, por lo que se decidió eliminar los reactivos de dicho factor. Finalmente, en la cuarta ronda resultaron tres factores que explican el $73,7 \%$ de la varianza común. El primer factor se nombró Inutilidad de tratamiento, e incluye tres reactivos que se refieren a que no importa el momento del diagnóstico ni el tratamiento que se siga, de todos modos el paciente morirá. El segundo factor denominado Predestinación, incluye dos reactivos que se refieren a la idea de que la enfermedad ya estaba establecida por fuerzas externas al individuo. El tercer factor llamado Pensamientos de muerte incluye tres reactivos que se refieren a la creencia de que el cáncer provoca la muerte de quien lo padece.

En la tabla 2 se presenta la matriz rotada con la saturación factorial de los ocho reactivos finales, distribuidos en los tres factores. En la misma tabla se muestran 
las alfas de Cronbach de cada uno de los factores, observándose que en el factor Predestinación la confiabilidad fue muy alta, en el de Inutilidad del tratamiento fue alta, y en el de Pensamientos de muerte fue aceptable. La confiabilidad del cuestionario global fue alta $(\alpha=0,80)$.

Tabla 2. Estructura factorial del IFPE-C en pacientes con cáncer de mama.

\begin{tabular}{|c|c|c|c|}
\hline Reactivo & $\begin{array}{l}\text { Inutilidad del } \\
\text { tratamiento } \\
\alpha=0,85\end{array}$ & $\begin{array}{l}\text { Predestinación } \\
\alpha=0,918\end{array}$ & $\begin{array}{l}\text { Pensamientos } \\
\text { de muerte } \\
\alpha=0,73\end{array}$ \\
\hline $\begin{array}{l}\text { Yo pienso que si a una persona le va a dar } \\
\text { cáncer, le va a dar no importa lo que haga. }\end{array}$ & &,- 867 & \\
\hline $\begin{array}{l}\text { Yo pienso que si a una persona le da cán- } \\
\text { cer, así le tocaba. }\end{array}$ & &,- 956 & \\
\hline $\begin{array}{l}\text { Yo pienso que si a una persona le toca que } \\
\text { le de cáncer, le va a dar cáncer. }\end{array}$ & &,- 843 & \\
\hline $\begin{array}{l}\text { Yo pienso que si a una persona le da cán- } \\
\text { cer, esa persona se va a morir pronto. }\end{array}$ & & & ,994 \\
\hline $\begin{array}{l}\text { Yo pienso que si le da cáncer a una per- } \\
\text { sona, ese es el modo del cual le tocaba } \\
\text { morirse. }\end{array}$ & & & ,620 \\
\hline $\begin{array}{l}\text { Yo pienso que si alguien tiene cáncer, no } \\
\text { importa si se lo encuentran temprano o } \\
\text { tarde, porque de todos modos va a morir de } \\
\text { cáncer. }\end{array}$ &, 755 & & \\
\hline $\begin{array}{l}\text { Yo pienso que si alguien tiene cáncer y } \\
\text { recibe tratamiento para curarse, de todas } \\
\text { maneras se va a morir de esta } \\
\text { enfermedad. }\end{array}$ & ,924 & & \\
\hline $\begin{array}{l}\text { Yo pienso que el cáncer matará a una per- } \\
\text { sona, no importa cuándo lo encuentren o } \\
\text { cómo lo curen. }\end{array}$ & ,789 & & \\
\hline
\end{tabular}

Método de extracción: Mínimos cuadrados no ponderados. Método de rotación: Oblimin con Kaiser.

En los análisis de correlaciones se observaron los siguientes resultados significativos: El factor Predestinación mostró correlaciones positivas con los factores de Inutilidad de tratamiento $(\mathrm{r}=0,21)$ y el de Pensamientos de muerte $(0,24)$. Así mismo, éste último correlacionó positivamente con el factor Inutilidad del tratamiento $(\mathrm{r}=0,46)$. Cabe aclarar que aunque las correlaciones observadas resultan ser bajas o moderadas, todas ellas tuvieron una $p<0,01$. 
Por último, al calcular los puntos de corte se encontró que el puntaje de 0 puede ser considerado ausencia de fatalismo, el puntaje de 1 a 3 fatalismo moderado, y el puntaje de 4 a 8 fatalismo elevado.

\section{Discusión}

El objetivo de la investigación fue validar el SPFI en población mexicana diagnosticada con cáncer. La escala resultante, el Inventario de Fatalismo de Powe en Español-cáncer (IFPE-C) posee propiedades psicométricas adecuadas, así como una estructura factorial clara e interpretable. El análisis factorial indicó la existencia de tres factores: Predestinación, Pensamientos de muerte, e Inutilidad del tratamiento. A pesar de que el IFPE-C posee una extensión y estructura factorial mas pequeña que el PFI y el SPFI, es decir, un menor numero de reactivos y de factores, logró explicar un mayor porcentaje de varianza. Además, los factores resultantes presentan congruencia teórica con el constructo Fatalismo en población ya diagnosticada con cáncer, esto debido a que los reactivos se agruparon de forma distinta a las dos versiones anteriores, un ejemplo de esto es que en la nueva estructura factorial se identificó un factor especifico para explorar la utilidad del tratamiento oncológico, cualidad que no poseen las versiones anteriores ${ }^{(6,22)}$. Cabe señalar que los tres factores fueron renombrados con la finalidad de establecer una interpretación clara de los reactivos con respecto al constructo Fatalismo en pacientes oncológicos.

Con respecto a la consistencia interna del instrumento, se observa que el coeficiente de Alfa de Cronbach en la escala global resultó ser similar al del PFI y SPFI. Sin embargo, a pesar de que las dos versiones anteriores tuvieron una estructura de cuatro factores, la consistencia interna de éstos no está reportada ${ }^{(6,22)}$. Por tal motivo, no se pudieron realizar comparaciones de la confiabilidad, pero cabe mencionar que ésta fue adecuada en el IFPE-C.

El IFPE-C puede ser usado con fines de investigación para ayudar a comprender los procesos psicológicos que dificultan la adecuada adaptación al cáncer, desde el diagnóstico hasta la supervivencia. Una de las variables con las que se podría relacionar es el afrontamiento, ya que existen algunas estrategias de afrontamiento que limitan la adaptación al diagnóstico o tratamiento del cáncer ${ }^{(28,29,)}$, y el fatalismo podría considerarse una barrera para el uso de estrategias de afrontamiento adecuadas ante la enfermedad. Otras de las variables que se podrían analizar junto con IFPE-C pueden serla angustia, la ansiedad y el distrés, pues algunos diagnósticos de cáncer generan angustia en los pacientes ${ }^{(30)}$, lo cual pudiera estar relacionado con el tipo de creencias que éstos tienen sobre la enfermedad; además, las creencias fatalistas desencadenan sensaciones de distrés y ansiedad con respecto al diagnóstico de cáncer en personas que no padecen esta enfermedad, ya que una persona con estas creencias tiende a asumir que el resultado de la enfermedad será negativo y algunas veces asociado con la muerte ${ }^{31}$.También sería recomendable analizar la relación de las creencias fatalistas con diferentes aspectos de la adherencia al tratamiento, pues las creencias tienen un impacto en las conductas de salud relacionadas con el cáncer ${ }^{(1,13,18)}$. Aunque cabe destacar que existe al menos una investigación en la que se utilizó el PFI para estudiar la relación entre creencias fatalistas y la adherencia a la quimioterapia, el estudio se llevó a cabo en Estados Unidos y no contempló pacientes de origen hispano ${ }^{(3)}$. 
El IFPE-C también podría ser utilizado en entornos clínicos, pues, como ya se mencionó, el fatalismo es una barrera para la adherencia a algunos tratamientos oncológicos como las quimioterapias ${ }^{(3)}$. Por lo tanto, la identificación de las creencias fatalistas en cada paciente, permitirá establecer un plan psicoterapéutico particular para fomentar la adherencia al tratamiento, así como la adaptación adecuada a los efectos colaterales de éste y a otras consecuencias adversas psicosociales del cáncer.

Por otro lado, cabe señalar que en esta investigación el IFPE-C fue validado solo con mujeres adultas con CaMa. Es indispensable que en investigaciones futuras sea validado con pacientes diagnosticados con diversos tipos de cáncer, tanto hombres como mujeres de diversas edades y estratos sociales, ya que las creencias fatalistas no son exclusivas de la población con CaMa. De hecho, como se mencionó en la Introducción, éstas forman parte de la cultura relacionada con la salud en la población latina $^{(5)}$. Además, también se sugiere, para futuras investigaciones, validar el IFPE-C para ser utilizado como un instrumento de tamizaje en población general en fase de detección oportuna, particularmente en personas con características que se han asociado a las creencias fatalistas ${ }^{(8,15)}$.

Finalmente, nuestros resultados deben ser interpretados en el contexto de sus limitaciones. En primer lugar, a pesar de que las participantes provenían de diversos estados del país, la muestra por conveniencia no es representativa de la población nacional; por lo tanto, estos hallazgos tienen una generalización limitada. En segundo lugar, el tamaño de la muestra a la que se tuvo acceso no permitió realizar el análisis confirmatorio de la estructura factorial ${ }^{(32)}$, por lo que es importante ampliar la muestra en futuras investigaciones. Finalmente, debido a que se requirió aplicar el cuestionario de forma oral para asegurar que las participantes comprendieran las preguntas, este hecho pudiera afectar la generalización de los resultados respecto a una aplicación por escrito.

A pesar de estas limitaciones, el IFPE-C demostró ser un instrumento valido y confiable, que puede ser utilizado tanto en entornos clínicos como de investigación para identificar las creencias fatalistas acerca del cáncer de los pacientes.

\section{Agradecimientos}

Agradecemos al Consejo Nacional de Ciencia y Tecnología por la beca de posgrado otorgada a Rosa Lilia Castillo López durante la realización de esta investigación (No. de becario 496877/284827)

\section{Referencias bibliográficas}

1. Marván ML, Ehrenzweig Y, Castillo-López RL. Fatalistic beliefs and cervical cancer screening among Mexican women. Health Care Women Int 2016;1-15. doi: 10.1080/07399332.2014.959169.

2. Leyva B, Allen JD, Tom LS, Ospino H, Torres MI, Abraido-Lanza AF. Religion, fatalism, and cancer control: a qualitative study among Hispanic Catholics. Am J Health Behav 2014;38:839. doi: 10.5993/AJHB.38.6.6. 
3. Wells JS, Strickland OL, Dalton JA, Freeman S. Adherence to intravenous chemotherapy in african-american and caucasian women with early stage breast cancer. Cancer Nurs 2015;38. doi: 10.1097/NCC.0000000000000139.

4. González P Nuñez A, Wang-Letzkus M, Lim J-W, Flores KF, Nápoles AM. Coping with breast cancer: reflections from chinese american, Korean american, and Mexicanamerican women. Health Psychol 35:19-28. doi: 10.1037/hea0000263.

5. Abraido-Lanza AF, Viladrich A, Flórez KR, Céspedes A, Aguirre AN, de la Cruz AA. Commentary: Fatalismo reconsidered: A cautionary note for health-related research and practice with Latino populations. Ethn Dis 2007;17:153-8.

6. Powe BD. Fatalism among elderly African Americans. Effects on colorectal cancer screening. Cancer Nurs 1995;18:385-92. doi: 10.1097/00002820-199510000-00008

7. Powe BD, Johnson A. Fatalism as a barrier to cancer screening among African-Americans: Philosophical perspectives. J Rel Health 1995;35:119-26. doi: 10.1007/BF02248767.

8. Befort CA, Nazir N, Engelman K, Choi W. Fatalistic cancer beliefs and information sources among rural and urban adults in the USA. J Cancer Educ 2013;28:521-6. doi: 10.1007/s13187-013-0496-7.

9. Cheng H, Sit JWH, Twinn SF, Cheng KKF, Thorne S. Coping with breast cancer survivorship in chinese women the role of fatalism or fatalistic voluntarism. Cancer Nurs 2013;36:236-44. doi: 10.1097/NCC.0b013e31826542b2.

10. Niederdeppe J, Levy AG. Fatalistic beliefs about cancer prevention and three prevention behaviors. Cancer Epidemiol Biomarkers Prev 2007;16:998-1103. doi: 10.1158/10559965.EPI-06-0608

11. Mc Mullin JM, Alba ID, Chavez LR, Hubbell FA. Influence of beliefs about cervical cáncer etiology on Paps mear use among Latina immigrants. Ethnic Health 2005;10:3-18. doi: 10.1080/1355785052000323001

12. Otero-Sabogal R, Stewart S, Sabogal F, Brown BA, Pérez-Stable EJ. Access and attitudinal factors related to breast and cervical cancer rescreening: why are Latinas still under screened? Health Educ Behav. 2003;337-59. doi: 10.1177/1090198103030003008

13. Espinosa K, Gallo LC. The relevance of fatalism in the study of Latinas' cancer screening behavior: A systematic review of the literature. Int J Behav Med 2011;18:310-8. doi: 10.1007/s12529-010-9119-4.

14. Shen L, Condit CM, Wright L. The psychometric property and validation of a fatalism scale. Psychol Health 2009;24:597-613. doi: 10.1080/08870440801902535.

15. Powe BD Finnie R. Cancer fatalism: The state of the science. Cancer Nurs 2003;26:45465. doi: 10.1097/00002820-200312000-00005

16. Miles A Voorwinden S Chapman S Wardle J. Psychologic predictors of cancer information avoidance among older adults: the role of cancer fear and fatalism. Cancer Epidemiol Biomarkers Prev. 2008;17:1872-9. doi: 10.1158/1055-9965.EPI-08-0074.

17. Kulakci H, Ayyildiz TK, Yildirim N, Ozturk O, Topan AK, Tasdemir N. Effects of breast cancer fatalism on breast cancer awareness among nursing students in Turkey. Asian Pac J Cancer Prev 2015;16:3565-72. doi: 10.7314/APJCP.2015.16.8.3565

18. Kulakci H, Kuzlu T, Veren F, Kose A. The effect of breast cancer fatalism on breast cancer awareness among turkish women. J Relig Health 2017;56:1537-52. doi: 10.1007/ s10943-016-0326-4.

19. Sheppard VB, Davis K, Boisvert M, Boisvert M, Jennings Y, Montalvo B. Do recently diagnosed black breast cancer patients find questions about cancer fatalism acceptable? A preliminary report. J Cancer Educ 2011;26:5-10. doi: 10.1007/s13187-010-0134-6. 
20. Gonzales FA, Hurtado de Mendoza A, Santoyo-Olsson J, Nápoles AM. Do coping strategies mediate the effects of emotional support on emotional well-being among Spanish-speaking Latina breast cancer survivors? Psychooncology. 2016;25:1286-92. doi: 10.1002/pon.3953.

21. Lagos VI, Perez MA, Ricker CN, Blazer KR, Santiago NM, Feldman N. Social-cognitive aspects of underserved Latinas preparing to undergo genetic cancer risk assessment for hereditary breast and ovarian cancer. Psychooncology 2008;17:774-82. doi: 10.1002/ pon.1358.

22. Lopez-McKee G, McNeill JA, Eriksen LR, Ortiz M. Spanish translation and cultural adaptation of the Powe Fatalism Inventory. J Nurs Scholarsh 2007;39:68-70. doi:10.1111/ j.1547-5069.2007.00145.x

23. Ferlay J, Soerjomataram I, Dikshit R, Eser S, Mathers C, Rebelo M, et al. Estimated agestandardized incidence rates (World) [Internet]. 2018. Disponible en: https://gco.iarc.fr/ today

24. Anastasi A, Urbina S. Psychological testing. 7a ed. New York: Prentice Hal; 2009. p.721

25. Ferrando PJ, Anguiano-Carrasco C. El análisis factorial como técnica de investigación en psicología. Papeles del psicólogo 2010; 31:1.

26. Beavers AS, Lounsbury JW, Richards JK, Huck SW, Skolits GJ, Esquivel S L. Practical considerations for using exploratory factor analysis in educational research. Practical Assessment, Research \& Evaluation 2013;18:6. http://pareonline.net/getvn.asp? $\mathrm{v}=18 \& \mathrm{n}=6$

27. Cronbach LJ, Shavelson RJ. My current thoughts on coefficient alpha and successor procedures. Educ Psychol Meas 2004;64:391-418. doi: 10.1177/0013164404266386

28. Carver CS. You want to measure coping but your protocol'too long: Consider the brief cope. Int J Behav Med 1997;4:92-100. doi: 10.1207/s15327558ijbm0401_6

29. Wang WT, Tu PC, Liu TJ, Yeh DC, Hsu WY. Mental adjustment at different phases in breast cancer trajectory: re-examination of factor structure of the Mini-MAC and its correlation with distress. Psychooncology 2013;22:768-74. doi: 10.1002/pon.3065

30. Dabrowski M, Boucher K, Ward JH, Lovell MM, Sandre A, Bloch J, et al. Clinical experience with the NCCN distress thermometer in breast cancer patients. J Natl Compr 2007;5:104-11. doi: 10.6004/jnccn.2007.0011

31. Bettencourt BA, Talley AE, Molix L, Schlegel R, Westgate SJ. Rural and urban breast cancer patients: Health locus of control and psychological adjustment. Psychooncology 2008;17:932-9. doi: 10.1002/pon.1315

32. Byrne BM. Structural equation modeling with AMOS: basic concepts, applications, and programming. 2a ed. New York: Taylor \& Francis; 2010. 
